(2) OPEN ACCESS

\section{Long-term effectiveness of faecal immunochemical test screening for proximal and distal colorectal cancers}

\author{
Han-Mo Chiu $\odot{ }^{1},{ }^{1}$ Grace Hsiao-Hsuan Jen, ${ }^{2}$ Ying-Wei Wang, ${ }^{3}$ Jean Ching-Yuan Fann, ${ }^{4}$ \\ Chen-Yang Hsu, ${ }^{2}$ Ya-Chung Jeng, ${ }^{5}$ Amy Ming-Fang Yen (ㄷ), \\ Sherry Yueh-Hsia Chiu (1) ${ }^{6}$ Sam Li-Sheng Chen (1), ${ }^{5}$ Wen-Feng Hsu, ${ }^{1,2}$ \\ Yi-Chia Lee (1), ${ }^{1}$ Ming-Shiang Wu (1), ${ }^{1}$ Chien-Yuan Wu, ${ }^{3}$ Yann-Yuh Jou, ${ }^{3}$ \\ Tony Hsiu-Hsi Chen ${ }^{2}$
}

- Additional material is published online only. To view, please visit the journal online (http://dx.doi.org/10.1136/ gutjnl-2020-322545).

For numbered affiliations see end of article.

\section{Correspondence to}

Dr Tony Hsiu-Hsi Chen, Institute of Epidemiology and Preventive Medicine, College of Public Health, National Taiwan University, Taipei 100, Taiwan; chenlin@ntu.edu.tw

Received 15 July 2020 Revised 8 December 2020 Accepted 3 January 2021 Published Online First 25 January 2021
Check for updates

(C) Author(s) (or their employer(s)) 2021. Re-use permitted under CC BY-NC. No commercial re-use. See rights and permissions. Published by BMJ.

To cite: Chiu H-M, Jen GH-H, Wang Y-W, et al. Gut

2021:70:2321-2329.

\section{ABSTRACT}

Objective To measure the effects of faecal immunochemical test (FIT) for colorectal cancer (CRC) screening on overall and site-specific long-term effectiveness of population-based organised service screening. Design A prospective cohort study of Taiwanese nationwide biennial FIT screening was performed. A total of 5417699 eligible subjects were invited to attend screening from 2004 through 2009 and were followed up until 2014. We estimated the adjusted relative rates (aRRs) on the effectiveness of reducing advanced-stage CRC (stage II+) and CRC death by Bayesian Poisson regression models with the full adjustment for a cascade of self-selection factors (including the screening rate and the colonoscopy rate) and the completeness of colonoscopy together with demographic features.

Results FIT screening (exposed vs unexposed) reduced the incidence of advanced-stage CRC (48.4 vs 75.7 per 100 000) and mortality (20.3 vs 41.3 per 100 000). Statistically significant reductions of both incidence of advanced-stage CRCs (aRR $=0.66,95 \% \mathrm{Cl} 0.63$ to 0.70 ) and deaths from CRC (aRR $=0.60,95 \%$ Cl 0.57 to 0.64) were noted. FIT screening was more effective in reducing distal advancedstage CRCs (aRR $=0.61,95 \% \mathrm{Cl} 0.58$ to 0.64 ) and CRC mortality ( $a R R=0.56,95 \% \mathrm{Cl} 0.53$ to 0.69 ) than proximal advanced CRCs (aRR=0.84, 95\% Cl 0.77 to 0.92 ) and CRC mortality (aRR=0.72, 95\% Cl 0.66 to 0.80).

Conclusion A large-scale population-based biennial FIT screening demonstrates 34\% significant reduction of advanced-stage CRCs and $40 \%$ reduction of death from CRC with larger long-term effectiveness in the distal colon than the proximal colon. Our findings provide a strong and consistent evidence-based policy for supporting a sustainable population-based FIT organised service screening worldwide. The disparity of site-specific long-term effectiveness also provides an insight into the remedy for lower effectiveness of FIT screening in the proximal colon.

\section{INTRODUCTION}

Colorectal cancer (CRC) is currently the fourth leading cause of cancer death worldwide, and screening is effective in reducing its incidence and mortality. ${ }^{1}$ While endoscopy-based screening is the

\section{Significance of this study}

What is already known on this subject?

- Faecal immunochemical test (FIT) has been reported to outperform guaiac faecal occult blood tests (gFOBT).

- The effectiveness of FIT in reducing colorectal cancer (CRC) mortality has been demonstrated recently by several population-based studies, but whether the similar benefits can be achieved for the proximal and distal colon still needs to be elucidated.

What are the new findings?

- A $60 \%$ screening rate in a large FIT service screening reduced the incidence of advancedstage CRC by $34 \%$ and CRC-related death by $40 \%$ after the full adjustment for self-selection factors and the quality of colonoscopy.

- Screening was more effective in reducing incidence of advanced-stage CRC and death from $C R C$ in the distal colon than the proximal colon.

How might it impact on clinical practice in the foreseeable future?

- The findings of long-term effectiveness provide consistent evidence-based policy for population-based FIT service screening worldwide.

- The disparity of site-specific effectiveness finds a clue to provide a remedy for improving the effectiveness of early detection of proximal CRCs from both aspects of screening polices and the quality of colonoscopy.

most effective method, stool-based screening tests specifically identify high-risk subjects for colonoscopy, rendering it the most popular screening test worldwide, especially in regions where the clinical capacity of endoscopy is limited. Faecal immunochemical test (FIT) has been reported to outperform guaiac faecal occult blood tests. ${ }^{2}$ The effectiveness of FIT in reducing CRC mortality 
has been demonstrated recently by several population-based studies. ${ }^{3-6}$

Despite these observational studies, there is an urgent need for health decision-makers worldwide to evaluate the longterm effectiveness of FIT screening in each large-scale organised service screening in order to ensure that its quality assurance is as good as that of these previous organised CRC screenings that have completely or partially used the FIT test and have already demonstrated the significant effectiveness of mortality reduction. ${ }^{4-6}$ So doing also provides an evidence-based policy for FIT screening with sustainability in order to meet the Sustainable Development Goal (SDG) 3 of global health. ${ }^{7}$ Our previous report on the results from the inaugural 5 years of the Taiwan Colorectal Cancer Screening Program with only one-fifth of screening rate has already demonstrated a significant $10 \%$ reduction in CRC mortality, almost attaining the performance of guaiac test with a longer follow-up time of more than 10 years. ${ }^{13}$ While our nationwide programme has been expanded to around $60 \%$ screening rate, it provides an opportunity to demonstrate whether the long-term effectiveness of population-based organised FIT service screening can be achieved like colonoscopy screening evaluated with a randomised trial design or a modelling approach. ${ }^{8-10}$

Moreover, as previous studies have demonstrated that FIT is less sensitive for detecting proximal advanced neoplasm than distal ones, ${ }^{11} 12$ whether the long-term effectiveness of FIT in reducing mortality from CRC and advanced-stage CRC also varies with anatomical site is therefore worthy of being investigated. To estimate the unbiased overall and site-specific effectiveness in population-based FIT service screening as if obtained in a randomised controlled trial with intention-to-treat (ITT) analysis, one has to make allowance for relevant factors pertaining to self-selection bias and incomplete colonoscopy.

In the present study, we aimed to elucidate whether and how the Taiwanese nationwide population-based FIT screening can lead to the reduction of overall and site-specific mortality and incident advanced-stage CRC by analysing a large-scale cohort eligible for screening in the inaugural 5 years of the programme with continuous biennial FIT screening with 10 years of follow-up. Such an evaluation would be based on a newly developed method for the full adjustment for relevant characteristics that affect self-selection bias and the completeness of colonoscopy.

\section{METHODS}

\section{Taiwan Colorectal Cancer Screening Program}

Taiwan Colorectal Cancer Screening Program is a nationwide population-based FIT service screening launched in 2004. The details of this screening programme have been described in full elsewhere. ${ }^{3}$ In brief, a biennial single-sample FIT screening has been provided universally for subjects aged 50-69 years since the inaugural period (2004-2009). In addition to age range, criteria also included whether the enrolled subject is free of alarming symptoms of CRC such as bowel habit change, defecation with mucous or passage of blood, and tenesmus, all of which were assessed by primary healthcare providers (physician, nurse and public health personnel). It is mandatory for them to obtain and report the signed record on the result of the eligibility assessment in written form. Information on the screened cohort has been centralised in our mass-screening registry. As massscreening registry and population registry have been stored as the centralised database in a nationwide scale, non-participants were ascertained after the linkage of the screened cohort from mass-screening registry with all eligible subjects recorded in population registry. During this period, people could only obtain the FIT kit at regional health centres prompted by the designated awareness campaign. To boost up the screening coverage, the government started to roll out the programme in 2010. People could also have the uptake of FIT screening in hospitals or clinics, in addition to the aforementioned sites, and the way of assessing eligibility was identical. ${ }^{13}$ One of two separate FIT kits (OC-SENSOR, Eiken Chemical Ltd, Tokyo, Japan, or HM-JACK, Kyowa Medex Co, Tokyo, Japan) was selected by each municipality or hospital/clinic according to its own purchasing process, both using $20 \mu \mathrm{g}$ of haemoglobin/g of faeces as the cut-off to determine positivity. People were notified of the FIT results by postal mail or by physician in the outpatient clinics, and those who had positive FITs were then referred for colonoscopy as a diagnostic examination within 3 months, and those who were considered as infeasible for colonoscopy or failed complete colonoscopy were then offered double-contrast barium enema as an alternative diagnostic procedure. This process was intensified by sending second or third notices via public health workers or nursing staff networks in the regional health centres, hospitals or clinics. A cascade of the entire screening process from uptake screening until diagnostic examination with complete colonoscopy to reach the cecum is diagrammed in figure 1 . All these relevant screening characteristics (such as the screening rate, the FIT positivity rate, the referral rate of diagnostic examinations, the rate of selecting colonoscopy as diagnostic examination, complete colonoscopy rate, and detection rates for advanced adenoma and cancers) of individual municipalities were regularly monitored using the central screening database in the Health Promotion Administration of the Taiwanese government. It should be noted that although these characteristics have improved with time (complete colonoscopy rate increased from $75 \%$ in 2004 until $96 \%$ in 2014), they have to be adjusted during the inaugural 5 years in order to estimate the unbiased effectiveness of FIT screening as close as to that estimated with a randomised controlled design while the self-selection bias and the completeness of colonoscopy are taken into account (see further). Those who had neoplasms detected and resected at colonoscopy were recommended for subsequent surveillance colonoscopy based on the initial findings as recommended by the current surveillance guidelines, and those who had negative examination were advised to receive biennial FIT screening within the programme. The FIT laboratories and endoscopic units are periodically audited and accredited by the screening organiser, which is described in our previous publications. ${ }^{14} 15$

\section{Study design, population and data collection}

In this prospective cohort study, 5417699 subjects who were considered as eligible for CRC screening during the inaugural 5 years (2004-2009) were continuously offered the subsequent screen on biennial basis and followed up until the end of 2014 (figure 2). ${ }^{3}$ Participants who underwent at least one FIT screening during the period of 2004-2014 constituted the exposed group, and the rest of the population formed the unexposed group. Note that whether the exposed group was further referred to have diagnostic examination and whether to have complete colonoscopy would be refined according to figure 1 , when allowance was made for self-selection bias and the quality of complete colonoscopy. Events were defined as incident advanced-stage (stage II+) CRC and CRC deaths. The screening database was linked to the national cancer registry and national death registry, from which the two aforementioned events could 
$r_{E}$ : Screening rate

$r_{P O S}$ : Positivity rate

$r_{R E F}$ : Diagnostic exam rate

$r_{C P}$ : Colonoscopy rate

$r_{C I}$ : Cecal intubation rate

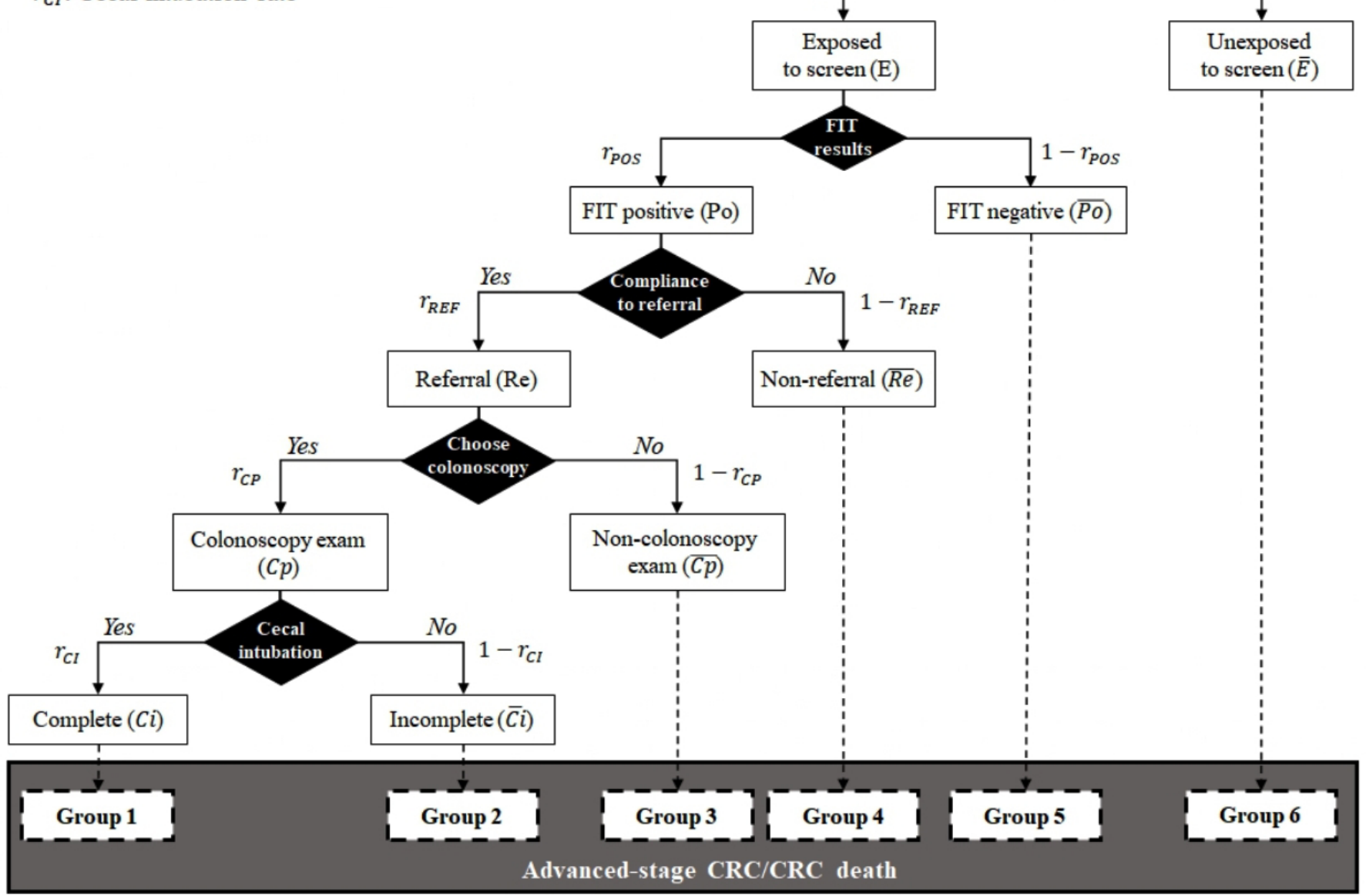

Figure 1 Cascade of screening indicators from the uptake of screening until the final confirmatory diagnosis with complete colonoscopy to reach the cecum for subjects invited to a population-based service screening programme for CRC. CRC, colorectal cancer; FIT, faecal immunochemical test.

be ascertained and verified. The coverage rate of the national cancer registry was reported to be $98.6 \%$ with accuracy of greater than 99\%. ${ }^{16}$ Both incident CRCs and CRC deaths were ascertained with the follow-up until the end of 2014. CRCs were

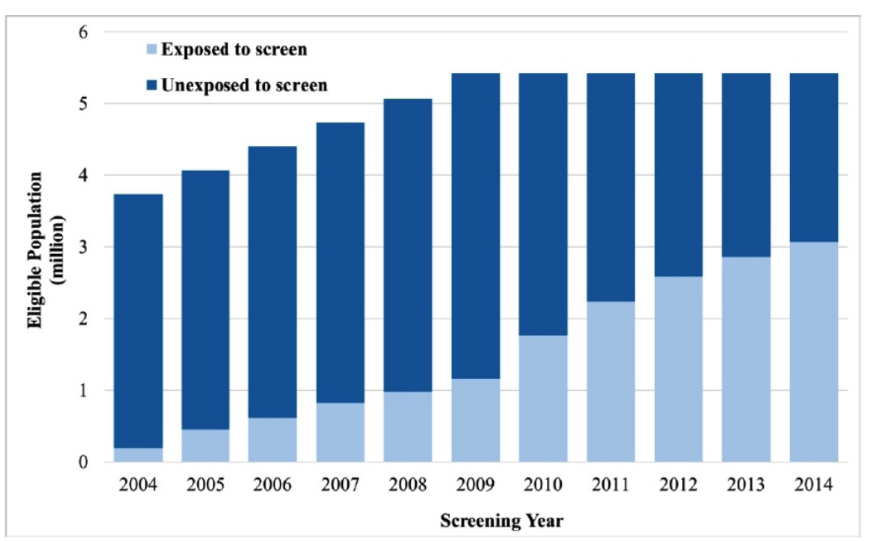

Figure 2 Schematic of the prospective cohort with the staggered entry to attend the first screen for the eligible population aged 50-69 years. staged in the light of the sixth or seventh version of the American Joint Committee on Cancer staging system. Colon anatomical site above the level of splenic flexure is defined as the proximal colon and the remaining segments and rectum as the distal colon.

\section{Statistical analysis}

Descriptive results for FIT positivity rate, diagnostic examination rate and detection rate for CRC are reported as percentages. The anatomical site and stage distribution of CRCs were identified in the exposed and the unexposed groups during the study period. FIT interval cancers were CRCs occurring within 2 years after negative FIT were defined, which enables us to calculate the programme sensitivity by anatomical site. ${ }^{15}$ Colonoscopy interval cancers refer to the incident CRCs that become symptomatic and diagnosed within the recommended surveillance interval after the baseline colonoscopy without the diagnosis of CRC. Incidence and mortality rates were expressed as events per $10^{5}$ person-years. Because it is a population-based service screening, subjects were enrolled by a gradual rolling-out strategy that constitutes a prospective cohort with the staggered entry to screen in different calendar years for all eligible Taiwanese population aged 50-69 years as shown in figure 2. This also means that the screening rate increased with year, although the overall 
screening rate was up to $57 \%$ until 2014 . The calculation of person-years for each eligible individual was therefore based on the follow-up time from the date of the starting year (2004) until the end of follow-up, which was defined as the occurrence of an event, death from competing causes, age of 69 years or the end of the study period, whichever came earlier. To classify person-years into two groups, the exposed group (attender) and the unexposed group (non-attender), individual variation with time to attend the first screen (time-dependent property) should be considered, namely, those who had been unexposed to screen since 2004 but attended FIT screening later during the study period contributed their person-years to the unexposed group prior to attend FIT screening, but person-years thereafter were attributed to the exposed group. Such a dynamic change of person-years belonging to the two groups due to the eligible subjects attending the first screen in a staggered entry is considered in the following Bayesian Poisson regression model for correcting self-selection bias with adjustment for the screening rate in a time-dependent manner. The same calculation of person-years accompanied with the exposure status to attend the screen was also applied to more than two groups further classified by referral versus non-referral to diagnostic examination, colonoscopy versus non-colonoscopy diagnostic examination, and complete versus incomplete colonoscopy while the following full adjustment for correcting self-selection bias and the completeness of colonoscopy was made.

\section{Self-selection bias adjustment with the screening rate}

As the screening rate of population-based service screening with a staggered entry as mentioned previously is a reflection of aggregated self-selection on population level, it is necessary to estimate unbiased effectiveness between the exposed group and the unexposed group like the comparison between the invited and the uninvited group in the absence of screening using the language of a randomised controlled trial design with ITT analysis. This is exemplified in online supplemental materials (see the online supplemental equation (S-1)). The unbiased relative risk for measuring the reduction of CRC death as a result of FIT screening is expressed as

$$
\frac{\mathrm{P}(\text { CRC Death (D) } \mid \text { Invited }(\mathrm{I}))}{\mathrm{P}(\text { CRC Death }(\mathrm{D}) \mid \text { Uninvited }(\overline{\mathrm{I}}))}
$$

To approximate this unbiased estimate on the population level, we have to correct self-selection bias with adjustment for the screening rate extending the method that has been developed $^{1718}$ and widely used in the evaluation of the effectiveness of a population-based organised service screening programme. ${ }^{31920}$ The risk of being dead from CRC as in the numerator of the equation (1) can be decomposed into the exposed group and the unexposed group pursuant to ITT analysis. Note that relative risk is changed to relative rate when person-year is used for the denominator. Take the primary endpoint of CRC mortality for example, the first adjusted relative rate $\left(a R_{1}\right)$ in relation to the screening rate can be derived with the following expression:

$$
\left.\mathrm{aRR}_{1} \cong \text { (screening rate }\right) \times \mathrm{RR}_{\mathrm{E}}+(1 \text { - screening rate }) \times \mathrm{RR}_{\overline{\mathrm{E}}}
$$

where $\mathrm{RR}_{\mathrm{E}}$ is the mortality rate of CRC in the exposed group compared with the mortality rate in the uninvited group, and $\mathrm{RR}_{\overline{\mathrm{E}}}$ is the mortality rate of CRC in the unexposed group compared with the mortality rate in the uninvited group. The two relative comparisons, the exposed vs the uninvited group $\left(R_{E}\right)$ and the unexposed group vs the uninvited group $\left(R_{\bar{E}}\right.$ ), are weighted by the screening rate. Note that the uninvited group (theoretically used in a randomised control design) can be approximated by using the pre-screening period with adjustment for an increasing growth rate (see below). The details of derivation are given in online supplemental materials.

Correction of self-selection bias with full adjustment

As self-selection bias can also be affected by the referral rate of diagnostic examinations among FIT positives and the choice of colonoscopy (colonoscopy rate) as diagnostic examination, and the quality of colonoscopy regarding the completeness of colonoscopy (cecal intubation rate), particularly in the inaugural period, the equation (2) following the equation (1) is further expanded to get the second adjusted relative rate $\left(\mathrm{aRR}_{2}\right)$ with the full adjustment for a cascade of these factors with the following expression:

$$
\begin{aligned}
& \mathrm{aRR}_{2} \cong \mathrm{RR}_{\mathrm{Ci}} \times(\text { Part I })+\mathrm{RR}_{\overline{\mathrm{Ci}}} \times(\text { Part II })+\mathrm{RR}_{\overline{\mathrm{Cp}}} \times(\text { Part III })+ \\
& \mathrm{RR}_{\overline{\mathrm{Re}}} \times(\text { Part IV })+\mathrm{RR}_{\overline{\mathrm{Po}}} \times(\text { Part V })+\mathrm{RR}_{\overline{\mathrm{E}}} \times\left(1-\mathrm{r}_{\mathrm{E}}\right)
\end{aligned}
$$

where

$$
\begin{aligned}
& (\text { Part I })=(\text { Cecal intubation rate }) \times(\text { Colonoscopy rate }) \times \\
& (\text { Referral rate }) \times(\text { Positive rate }) \times(\text { Screening rate }) \\
& (\text { Part II })=(1-\text { Cecal intubation rate }) \times(\text { Colonoscopy rate }) \times \\
& (\text { Referral rate }) \times(\text { Positive rate }) \times(\text { Screening rate }) \\
& (\text { Part III })=(1-\text { Colonoscopy rate }) \times(\text { Referral rate }) \times \\
& (\text { Positive rate }) \times(\text { Screening rate }) \\
& (\text { Part IV })=(1-\text { Referral rate }) \times(\text { Positive rate }) \times(\text { Screening rate }) \\
& (\text { Part V) }=(1-\text { Positive rate }) \times(\text { Screening rate })
\end{aligned}
$$

$\mathrm{RR}_{\mathrm{Ci}}, \mathrm{RR}_{\overline{\mathrm{Ci}}}, \mathrm{RR}_{\overline{\mathrm{Cp}}}, \mathrm{RR}_{\overline{\mathrm{Re}}}$ and $\mathrm{RR}_{\overline{\mathrm{Po}}}$ represent relative rates for a series of relative comparisons, including those with complete colonoscopy (successful cecal intubation), incomplete colonoscopy, not selecting colonoscopy as diagnostic examination, refusing diagnostic examination and negative FIT results compared with the uninvited group as indicated in equation (1). All these relative rates are weighted by a cascade of screening characteristics affecting the performance of the screening process denoted by $r_{E}$ for the exposed group, $r_{P O s}$ for positive FIT test, $r_{\mathrm{REF}}$ for the referral rate for diagnostic examination, $\mathrm{r}_{\mathrm{CP}}$ for the colonoscopic rate and $r_{C I}$ for the rate of completing colonoscopy with a reach to caecum. The derivation of equation (3) is elaborated in the online supplemental materials.

\section{Bayesian Poisson regression model}

To make the two adjusted relative rates (aRRs) amenable to estimation, we exploited the Bayesian Poisson regression model with time-varying person-years based on equation (2) and also online supplemental equation (S-3) in the online supplemental materials, with adjustment for the screening rate alone written as follows:

$$
\begin{aligned}
& \log (\text { expected number of advanced }- \text { stage CRC or death from CRC }) \\
& =\log (\text { time }- \text { depdent person }- \text { years })+\beta_{\mathrm{b}} \\
& +\beta_{1} \cdot(\text { exposed })+\beta_{2} \cdot(\text { unexposed })+\beta_{3} \cdot(\text { age })+\beta_{4} \cdot(\text { gender })
\end{aligned}
$$

where $\beta_{\mathrm{b}}(0.0443=\log (1.045))$ is the natural growth rate of incident trend of $\mathrm{CRC}$ as indicated earlier. The Bayesian Poisson regression model estimated this aRR through two relative rates $\left(R_{E}\right.$ and $\mathrm{RR}_{\overline{\mathrm{E}}}$ ) as indicated previously in terms of the exponent of two regression coefficients, $\beta_{1}$ and $\beta_{2}$, obtained by modelling the relationship of the status of screen $(1=$ exposed for attenders, $0=$ unexposed for non-attenders) represented by two indicator functions to the number of CRC death.

Equation (4) takes into account extraneous factors, age, gender and increasing incidence rate, that have been applied previously. ${ }^{18} 19$ In Taiwan, as incidence of CRC is increasing annually, $4.5 \%$ of the growth rate of biologically increasing incidence rate is therefore 
required to make adjustment by extrapolation based on data from 1998 to 2003 obtained from the national cancer registry before the launch of screening. Thus, the annual mortality of 50.2 per 100000 between 1998 and 2003 was adjusted to 52.5 per 100 000, which is taken as the expected mortality in the absence of mass screening that is supposed to be equivalent to the uninvited (control) group of a randomised controlled trial for self-selection bias adjustment.

Regarding the full adjustment for self-selection bias and the completeness of colonoscopy, the corresponding Bayesian Poisson regression model expanded from equation (3) is expressed as

$$
\begin{aligned}
& \log (\text { expected number of advanced }- \text { stage CRC or death fromCRC }) \\
& =\log (\text { time }- \text { depdent person }- \text { years })+\beta_{\mathrm{b}} \\
& +\beta_{1} \cdot(\text { Complete colonoscopy })+\beta_{2} \cdot(\text { Incomplete colonoscopy }) \\
& +\beta_{3} \cdot(\text { Non }- \text { colonoscop exam })+\beta_{4} \cdot(\text { Non }- \text { referral }) \\
& +\beta_{5} \cdot(\text { FIT }- \text { negative })+\beta_{6} \cdot(\text { unexposed })+\beta_{7} \cdot(\text { age }) \\
& +\beta_{8} \cdot(\text { gender })
\end{aligned}
$$

Recall that the distinction of the Bayesian Poisson regression model shown in equation (5) different from that in the equation (4) is that the equation (5) further divides the exposed group in the equation (4) according to a cascade of factors after the exposure to screen (including positive FIT, referral rate, the choice of colonoscopy and complete colonoscopy), as shown in figure 1 with the corresponding coefficients from $\beta_{1}$ to $\beta_{5}$. The details of the derivation are also elaborated in the online supplemental materials.

The mortality of CRC and the incidence of advanced-stage CRC reduction after adjustment was calculated as $\left(1-\mathrm{aRR}_{1}\right.$ or 2 )$\times 100 \%$.

\section{Patient and public involvement}

It was not appropriate or possible to involve patients or the public in the design, conduction, reporting or dissemination plans of our research.

\section{RESULTS}

Of 5417699 subjects aged 50-69 years enrolled in our prospective cohort as mentioned previously, a total of 3067853 subjects participated in at least one FIT screening, and 2349846 subjects did not receive any FIT screening, yielding a screening coverage rate of 56.6\%. Among screened subjects, 1605200 participated in two or more rounds of FIT screening, indicating a repeat screening rate of $52.3 \%$. The positivity rate of FIT was $7.0 \%$ in the first round and $6.4 \%$ in the subsequent rounds. A total of $70.0 \%$ of FIT posi-

\begin{tabular}{|c|c|c|}
\hline & $\begin{array}{l}\text { Unexposed group } \\
(\mathrm{N}=2349846)\end{array}$ & $\begin{array}{l}\text { Exposed group } \\
(\mathrm{N}=3067853)\end{array}$ \\
\hline \multicolumn{3}{|l|}{ Sex } \\
\hline Male, n (\%) & $1184249(50.4)$ & $1374146(44.8)$ \\
\hline Female, $n(\%)$ & 1165597 (49.6) & 1693707 (55.2) \\
\hline Subsequent screening, $\mathrm{n}(\%)$ & - & $1605200(52.3)$ \\
\hline Incident CRC, $\mathrm{n}$ & 36018 & 13619 \\
\hline CRC death, $\mathrm{n}$ & 15550 & 3077 \\
\hline
\end{tabular}
tive subjects underwent diagnostic exams (89.8\% with colonoscopy) in the first round and $62.5 \%$ in the subsequent rounds $(94.1 \%$ with colonoscopy) (table 1). A total of 6756 and 3118 CRCs were detected in the first and subsequent screening rounds, respectively, yielding a detection rate of 2.20 and 1.94 per 1000 FITs.

CRC, colorectal cancer.
Cancer stage distribution between screened and unscreened groups

The proportions of CRC identified in the exposed group and the unexposed group at each stage within the study period are shown in table 2 . The rate of incomplete cancer stage information in detected cancers was $10.8 \%$ in the exposed group and $15.9 \%$ in the unexposed group.

\section{Incidence of advanced-stage CRC after FIT screening}

Table 3 shows the incidence rate of advanced-stage CRC, which was 48.4 and 75.7 per $10^{5}$ person-years in the exposed and the unexposed groups, respectively. The crude relative rate (cRR) of incidence of advanced-stage CRC was 0.64 (95\% CI 0.62 to 0.66). tTable 3 also shows after adjustment for age, gender and the screening rate in relation to self-selection bias, using equation (2) and the corresponding regression equation (4), the $\mathrm{aRR}_{1}$ of reducing incident advanced-stage CRCs was 0.71 (95\% CI 0.68 to 0.75$)$. Further adjustment for referral and completeness of colonoscopy using equations (3) and (5) gave 0.66 (95\% CI 0.63 to 0.70 ) of the $\mathrm{aRR}_{2}$ of reducing incident advanced-stage CRCs.

\section{CRC mortality after FIT screening}

Table 3 shows CRC mortality was 20.3 and 41.3 per $10^{5}$ personyears in the exposed and the unexposed groups, respectively. The cRR of CRC deaths was 0.49 (95\% CI 0.47 to 0.51 ). After adjusting for age, gender and the screening rate related to selfselection bias, the $\mathrm{aRR}_{1}$ of reducing death from CRC was 0.65 (95\% CI 0.62 to 0.69). The full adjustment for selection bias and completeness of colonoscopy involving the performance of a cascade of screening process led to an estimate of 0.60 (95\% CI 0.57 to 0.64 ) of the $\mathrm{aRR}_{2}$ of reducing death from CRC.

\section{Incidence of advanced-stage CRC and mortality by anatomical sites}

Table 3 also shows the $\mathrm{aRR}_{1}$ values for the reduction of sitespecific incidence of advanced-stage CRCs with only adjustment for the screening rate were 0.92 (95\% CI 0.84 to 1.00 ) and 0.65 (95\% CI 0.62 to 0.69$)$ for the proximal and the distal colon, respectively. After the full adjustment, the $\mathrm{aRR}_{2}$ values of reducing incident advanced-stage CRC were 0.84 (95\% CI 0.77 to 0.92 ) and 0.61 (95\% CI 0.58 to 0.64 ) for the proximal and the distal colon, respectively. The corresponding $\mathrm{ARR}_{1}$ values for the reduction of CRC mortality with only adjustment for the screening rate were 0.79 (95\% CI 0.72 to 0.87$)$ and $0.61(95 \%$ CI 0.57 to 0.64 ), respectively. The counterparts of reducing CRC mortality with the full adjustment were $0.72(95 \% \mathrm{CI}$ 0.66 to 0.80 ) and 0.56 (95\% CI 0.53 to 0.59$)$, respectively. It is obvious that the incremental effectiveness was larger in the proximal colon ( $8 \%$ for incidence of advanced-stage CRC and $7 \%$ for CRC mortality) than in the distal colon (4\% for incidence of advanced-stage CRC and 5\% for CRC mortality) after full adjustment from the screening rate to the completeness of colonoscopy.

The sensitivity analysis also shows that the $a R_{2}$ was further reduced to 0.64 (95\% CI 0.61 to 0.67$)$ for incident advanced CRCs and 0.57 (95\% CI 0.54 to 0.60 ) for CRC mortality provided that $100 \%$ completeness of colonoscopy can be achieved.

\section{Site-specific accuracy of FIT}

The disparity of site-specific finding on effectiveness was also supported by the difference in site-specific sensitivity with the order 
Table 2 Distribution of CRC stages in the exposed and unexposed groups and interval cancer diagnosed within the study cohort

\begin{tabular}{|c|c|c|c|c|c|c|c|c|}
\hline \multirow[b]{3}{*}{ AJCC staging* } & \multirow{2}{*}{\multicolumn{2}{|c|}{ Unexposed group }} & \multicolumn{6}{|c|}{ Exposed group } \\
\hline & & & \multicolumn{2}{|c|}{ Overallt } & \multicolumn{2}{|c|}{ Only attended first FIT screening } & \multicolumn{2}{|c|}{$\begin{array}{l}\text { Attended subsequent FIT } \\
\text { screening }\end{array}$} \\
\hline & $\mathrm{N}$ & $\%$ & N & $\%$ & N & $\%$ & N & $\%$ \\
\hline \multicolumn{9}{|l|}{ Overall } \\
\hline 0 & 2019 & 6.7 & 4832 & 22.3 & 2024 & 21.5 & 1544 & 29.6 \\
\hline 1 & 4587 & 15.1 & 5753 & 26.6 & 2677 & 28.5 & 1591 & 30.5 \\
\hline II & 7317 & 24.2 & 3585 & 16.5 & 1594 & 17.0 & 680 & 13.1 \\
\hline III & 8812 & 29.1 & 4962 & 22.9 & 2195 & 23.4 & 1037 & 19.9 \\
\hline IV & 7560 & 25.0 & 2533 & 11.7 & 910 & 9.7 & 358 & 6.9 \\
\hline Total & 30295 & 100.0 & 21665 & 100.0 & 9400 & 100.0 & 5210 & 100.0 \\
\hline \multicolumn{9}{|l|}{ Distal } \\
\hline 0 & 1593 & 7.0 & 3665 & 23.0 & 1625 & 22.4 & 1153 & 31.2 \\
\hline I & 3919 & 17.1 & 4524 & 28.4 & 2152 & 29.7 & 1180 & 32.0 \\
\hline II & 5170 & 22.6 & 2346 & 14.7 & 1117 & 15.4 & 398 & 10.8 \\
\hline III & 6747 & 29.4 & 3699 & 23.2 & 1711 & 23.6 & 734 & 19.9 \\
\hline IV & 5487 & 23.9 & 1699 & 10.7 & 649 & 8.9 & 225 & 6.1 \\
\hline Total & 22916 & 100.0 & 15933 & 100.0 & 7254 & 100.0 & 3690 & 100.0 \\
\hline \multicolumn{9}{|l|}{ Proximal } \\
\hline 0 & 410 & 5.7 & 1160 & 20.5 & 397 & 18.6 & 389 & 25.8 \\
\hline I & 638 & 8.9 & 1211 & 21.4 & 522 & 24.5 & 409 & 27.1 \\
\hline II & 2076 & 28.9 & 1205 & 21.3 & 469 & 22.0 & 278 & 18.4 \\
\hline III & 2006 & 28.0 & 1249 & 22.1 & 483 & 22.7 & 300 & 19.9 \\
\hline IV & 2045 & 28.5 & 827 & 14.6 & 259 & 12.2 & 132 & 8.8 \\
\hline Total & 7175 & 100.0 & 5652 & 100.0 & 2130 & 100.0 & 1508 & 100.0 \\
\hline
\end{tabular}

*Staging was based on AJCC sixth version before 2010 and seventh version thereafter. Stage information was incomplete in $10.8 \%$ in the screened group and $15.9 \%$ in unscreened group.

†Also includes FIT interval cancer, colonoscopy interval cancer and CRC of non-compliance with colonoscopy, in addition to CRC detected at first and subsequent screening rounds of next two columns.

AJCC, American Joint Committee on Cancer; CRC, colorectal cancer; FIT, faecal immunochemical test.

of $62.9 \%$ for proximal cancers $(72.7 \%$ for advanced stage ones) and $73 \%$ for distal cancers (83.2\% for advanced stage ones).

\section{DISCUSSION}

This is the first study with the evaluation of long-term effectiveness of population-based FIT service screening based on the full adjustment for a cascade of self-selection factors from screening uptake, the confirmatory exam rate, to the choice of colonoscopy and also the allowance made for the completeness of colonoscopy. The results not only demonstrate the overall statistically significant reduction of incidence of advanced-stage CRC (34\%) and mortality for CRC (40\%), given 10 years of follow-up, but also show larger effectiveness in the distal colon than in the proximal colon (39\% vs $16 \%$ for advanced-stage CRC and 44\% vs 28\% for CRC mortality), although the findings on both locations were still statistically significant.

\section{Causes for the disparity of site-specific long-term effectiveness}

The most important finding here is pertaining to the difference in site-specific long-term effectiveness of reducing incident advanced-stage CRCs and death from CRC resulting from FIT screening. There are two major causes that may be responsible for such a disparity, including the quality of colonoscopy, such as the completeness of colonoscopy and lower accuracy of FIT for detecting proximal neoplasms resulting from possibly different tumour biology of proximal and distal neoplasms and natural disease progression.
As far as the former is concerned, our site-specific findings found that larger effectiveness was gained for the proximal colon compared with the distal colon particularly when the allowance was made for the completeness of colonoscopy. This finding on the site-specific reduction of advanced CRC also supports the site-specific reduction in incidence of CRC in the Kaiser Permanente Northern California (KPNC) study, although the screening modality in their study consisted of guaiac fecal occult blood test (gFOBT) and sigmoidoscopy, outreaching FIT or colonoscopy. All these findings not only explain the contribution of incomplete colonoscopy to a smaller long-term effectiveness of reducing death from CRC in the proximal colon but also account for more proximal interval cancers after colonoscopy. Previous studies have also demonstrated that CRC occurring after colonoscopy was highly associated with inadequate colonoscopy quality in terms of low adenoma detection rate and failed cecal intubation. ${ }^{14-24}$ It is therefore possible that the colonoscopy for detecting CRCs may vary with anatomical sites.

There are several aspects related to the site-specific accuracy of FIT, resulting in the disparity of site-specific effectiveness. Frist of all, the detectability of FIT with 2 years of interscreening interval for the proximal cancers may be insufficient as the proximal CRCs may have a more rapid progression to severe CRC than the distal ones. This is supported by the fact that stage distribution was very different for proximal and distal CRC, which might in part account for the survival differences, and there were more proximal interval cancers. 
Table 3 Incidence of advanced CRCs or mortality from CRC in the exposed and unexposed groups and their crude and adjusted relative rates of the effectiveness of FIT screening between the two groups

\begin{tabular}{|c|c|c|c|c|c|c|c|c|}
\hline & \multicolumn{2}{|c|}{$\begin{array}{l}\text { Unexposed group } \\
(\mathrm{N}=2349846)\end{array}$} & \multicolumn{2}{|c|}{$\begin{array}{l}\text { Exposed group } \\
(\mathrm{N}=3067853)\end{array}$} & \multicolumn{4}{|c|}{ Relative rate $(95 \% \mathrm{CI})$, exposed versus unexposed } \\
\hline & Case (n) & $\begin{array}{l}\text { Rate } \\
\left(\text { per } 10^{5}\right)\end{array}$ & Case (n) & $\begin{array}{l}\text { Rate } \\
\left.\text { (per } 10^{5}\right)\end{array}$ & Crude & $\begin{array}{l}\text { Age-adjusted and } \\
\text { gender-adjusted }\end{array}$ & $\begin{array}{l}\text { aRR } \\
\text { (adjusting for screening rate)* }\end{array}$ & $\begin{array}{l}\text { aRR }_{2} \\
\text { (full adjustment)‡ }\end{array}$ \\
\hline \multicolumn{9}{|c|}{ Incidence of advanced cancert } \\
\hline Overall & 23689 & 75.7 & 6381 & 48.4 & $0.64(0.62$ to 0.66$)$ & $0.52(0.50$ to 0.53$)$ & $0.71(0.68$ to 0.75$)$ & $0.66(0.63$ to 0.70$)$ \\
\hline Proximal & 6127 & 19.6 & 2070 & 15.7 & $0.80(0.76$ to 0.84$)$ & $0.62(0.59$ to 0.65$)$ & $0.92(0.84$ to 1.00$)$ & $0.84(0.77$ to 0.92$)$ \\
\hline Distal & 17404 & 55.6 & 4267 & 32.3 & $0.58(0.56$ to 0.60$)$ & $0.48(0.46$ to 0.50$)$ & $0.65(0.62$ to 0.69$)$ & $0.61(0.58$ to 0.64$)$ \\
\hline \multicolumn{9}{|l|}{ Mortality } \\
\hline Overall & 15550 & 41.3 & 3077 & 20.3 & $0.49(0.47$ to 0.51$)$ & 0.44 (0.43 to 0.46$)$ & 0.65 (0.62 to 0.69$)$ & 0.60 (0.57 to 0.64$)$ \\
\hline Proximal & 4004 & 10.6 & 988 & 6.5 & 0.61 (0.57 to 0.66$)$ & $0.54(0.50$ to 0.58$)$ & $0.79(0.72$ to 0.87$)$ & 0.72 (0.66 to 0.80$)$ \\
\hline Distal & 11440 & 30.4 & 2076 & 13.7 & $0.45(0.43$ to 0.47$)$ & $0.41(0.39$ to 0.43$)$ & $0.61(0.57$ to 0.64$)$ & $0.56(0.53$ to 0.59$)$ \\
\hline
\end{tabular}

Person-years were 13196865 in the exposed group and 31294921 in the unexposed group for calculating incidence of advanced cancer; person-years was 15179449 in the exposed group and 37658371 in the unexposed group for calculating CRC mortality.

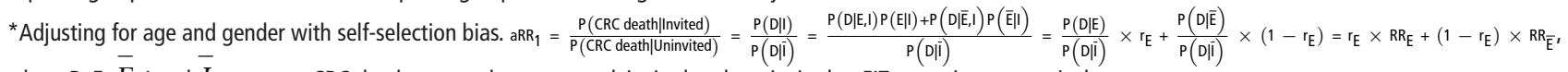
where $\mathrm{D}, \mathrm{E}, \bar{E}$, I and $I$ represent $\mathrm{CRC}$ death, exposed, not exposed, invited and not invited to FIT screening, respectively. tAdvanced cancer refers to CRC stage II or higher.

$\ddagger$ Adjusting for age, gender, attendance, referral, selection of diagnostic examination modality and completeness for colonoscopy, $(\mathrm{aRR} 2)=\frac{\mathrm{P}(\mathrm{CRCdeath} \mid \mathrm{Invited})}{\mathrm{P}(\mathrm{CRCdeath} \mid \mathrm{Uninvited})}$ with numerator $\mathrm{P}(\mathrm{CRC}$ death $\mid$ Invited $)=$

$\mathrm{P}($ CRC death $\mid$ Complete Colonoscopy $) \cdot \mathrm{r}_{\mathrm{CI}} \cdot \mathrm{r}_{\mathrm{CP}} \cdot \mathrm{r}_{\mathrm{REF}} \cdot \mathrm{r}_{\mathrm{POS}} \cdot \mathrm{r}_{\mathrm{E}}+$

$\mathrm{P}($ CRC death $\mid$ Incomplete Colonoscopy $) \cdot\left(1-\mathrm{r}_{\mathrm{CI}}\right) \cdot \mathrm{r}_{\mathrm{CP}} \cdot \mathrm{r}_{\mathrm{REF}} \cdot \mathrm{r}_{\mathrm{POS}} \cdot \mathrm{r}_{\mathrm{E}}+$

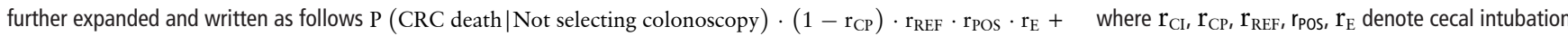

$\mathrm{P}(\mathrm{CRC}$ death $\mid$ Non - referral $) \cdot\left(1-\mathrm{r}_{\mathrm{REF}}\right) \cdot \mathrm{r}_{\mathrm{POS}} \cdot \mathrm{r}_{\mathrm{E}}+$

$\mathrm{P}($ CRC death $\mid$ Negative $) \cdot\left(1-\mathrm{r}_{\mathrm{POS}}\right) \cdot \mathrm{r}_{\mathrm{E}}+$

$\mathrm{P}($ CRC death $\mid$ Non - attender $) \cdot\left(1-\mathrm{r}_{\mathrm{E}}\right)$

rate, the proportion of selecting colonoscopy, referral rate to diagnosticexamination, positive rate and screening rate

$\mathrm{aRR}_{1}$, first adjusted relative rate; $\mathrm{ARR}_{2}$, second adjusted relative rate; CRC, colorectal cancer; FIT, faecal immunochemical test.

Second, CRCs even at the same stage with BRAF mutation (serrated pathway) were reported to have more grave outcome than CRC arisen via the so-called conventional pathway. ${ }^{25} 26$ Such a biological difference can also explain the difference in survival because serrated lesions are mainly located at proximal colon. Previous studies have also shown that FIT sensitivity was lower for proximal advanced neoplasms because sessile serrated adenoma/polyps or other non-polypoid neoplasms that are more likely to be missed by FIT or colonoscopy preponderate in the proximal location. ${ }^{11} 1227$

Third, it is also reasonable for a more advanced degree of haemoglobin degradation of blood originated from proximally located lesions during bowel passage. ${ }^{28}$ Given that adenomas bleed less than cancers, detection of proximal lesions is more likely to be compromised by degradation. As the removal of adenoma is what reduces incidence this issues of degradation is also very likely to explain the lesser effect on incidence and mortality of proximal cancers. Our site-specific sensitivity results on invasive CRCs also support FIT interval cancer, colonoscopy interval cancers, or CRC in those who were not compliant with diagnostic exams. ${ }^{1421-2429}$ The disparity of site-specific sensitivity estimates is consistent with the finding in a recent Italian study that proportional interval cancer rate was significantly higher in the proximal colon than in the distal colon and rectum. ${ }^{30}$

\section{Comparison with previous studies on FIT screening programme}

Based on the explanation on the completeness of colonoscopy responsible for the disparity of site-specific long-term effectiveness as indicated previously, it would not be surprised to find that after the correction for self-selection bias with the full adjustment, our results of reducing incidence of advancedstage CRC and long-term effectiveness of mortality reduction resulting from a population-based service screening were consistent with those the results of two previous studies from the Northern Italy FIT screening programme ${ }^{4}$ and the KPNC organised CRC screening programme with various screening modalities (including outreaching FIT) ${ }^{6}$ that are with high performance of the uptake of screening test and colonoscopy. Such a consistency implies that we would further expect an empirical finding on even larger reduction of CRC mortality by the continued Taiwanese nationwide FIT screening programme provided that these basic screening characteristics and the completeness of colonoscopy can be improved to a comparable level to the aforementioned FIT screening programmes. This again suggests high screening rate and the completeness of colonoscopy cannot be overemphasised in the implementation of a large populationbased service screening.

\section{Remedies for reducing the disparity of site-specific effectiveness}

In addition to providing a high-quality complete colonoscopy to reduce the disparity of site-specific interval cancer and longterm effectiveness of two-tier FIT screening, certain approaches can also be considered to reduce the disparity of site-specific FIT interval cancers resulting from the inaccuracy of FIT in the proximal colon. These include shortening interscreening intervals, increasing stool sample numbers and lowering the cutoff for determining FIT positivity. It should be also noted that these approaches may enhance the odds of detecting advanced 
adenoma and early CRC, but may increase the demand for colonoscopy and put further stress on the currently constrained colonoscopy capacity. ${ }^{31}$ Applying more sensitive tests such as the multitarget stool DNA test may be another viable approach to improve test sensitivity, but its high cost, uncertain or possibly low public acceptance, and unknown long-term effectiveness are obvious barriers for implementing it in a large-scale populationbased screen at the present time. ${ }^{32}$ Quality assurance of FIT and appropriate collection and management of stool sample is also indispensable for reducing the risk of FIT interval cancer, thereby maximising the effectiveness of FIT screening. Our previous study has demonstrated that different FIT kit might have different performance in terms of positive predictive value for CRC, leading to different risks of FIT interval cancers. Hence, the HM-JACK kits have been replaced with new generation HM-JACKarc in our programme. ${ }^{15}$

The strength of the current study is twofold. An innovative methodology was developed and applied to estimating the unbiased relative rate of two primary outcomes following ITT analysis with the full adjustment for a cascade of self-selection factors and the completeness of colonoscopy. Such a comprehensive adjustment that has never been done before not only renders the estimated effectiveness in population-based service screening as close as possible to the true value based on a randomised controlled trial design but also suggests that the quality of colonoscopy, more specifically, the completeness of colonoscopy, makes a contribution to the disparity of site-specific long-term effectiveness.

The present study is not without limitation. First, the diagnostic examination rate was unsatisfactory in Taiwanese programme. While the rate was about $80 \%$ in the inaugural 5 years of the programme as previously reported, it declined along with the rapid rolling out process since 2010 when FIT positive cases expanded by more than three times. ${ }^{3}{ }^{11} 33$ The similar findings of unsatisfactory referral rate for colonoscopy were also noted in two recent US studies. ${ }^{3435}$ Currently, the rate has gradually recovered to higher than $70 \%$ under the effort of the screening organiser, healthcare providers, and professional societies. Nevertheless, we have observed a significant reduction of CRC mortality and incidence of advanced-stage CRC, and once such a rate could be further improved, together with high screening rate and the colonoscopy quality, then we can expect an even larger magnitude of screening effectiveness. Second, we did not evaluate the overall incidence rate in our study as did in the KPNC study because, to a greater extent, the follow-up time in this study is still too short due to the staggered entry property of the current study and, to a lesser extent, still a lower screening rate and insufficiently high colonoscopy compliance rate with the early cohort (between 2004 and 2009) that is used for analysis. The reduction of overall incidence rate may require a longer follow-up period due to the long dwelling time from adenoma to invasive cancer. ${ }^{36}{ }^{37}$ One would anticipate a reduction of the incidence of CRC from the screening programme owing to the removal of adenoma. Mandel et al has demonstrated a $17 \%$ (95\% CI 6\% to 27\%) reduction with the biennial fecal occult blood test (FOBT) in the Minnesota trial. ${ }^{38}$ It could be underestimated with a relatively shorter follow-up time in the present study. Moreover, a longer follow-up time would also be required to deal with the lead-time issue in the early phase of service screening programme. Nonetheless, a comparably shorter dwelling time from early to advanced-stage cancer makes it possible to evaluate the reduction in advanced-stage cancer at an earlier timing as we did in the current study. The further follow-up of the cohort to elucidate the long-term effectiveness of reducing overall incidence is mandatory.
In conclusion, we demonstrated that FIT screening after a long-term follow-up is effective in reducing the risk of advancedstage CRC and its mortality, with effectiveness consistently stronger for distal CRCs compared with proximal ones. Our current results on long-term effectiveness, together with the findings from previous studies, may provide a strong and consistent evidence-based policy for supporting a sustainable populationbased FIT organised service screening worldwide, which is very meaningful for attaining the SDG 3 of global health.

\section{Author affiliations}

${ }^{1}$ Department of Internal Medicine, National Taiwan University Hospital, Taipei, Taiwan ${ }^{2}$ Institute of Epidemiology and Preventive Medicine, College of Public Health, National Taiwan University, Taipei, Taiwan

${ }^{3}$ Health Promotion Administration, Ministry of Health and Welfare, Taipei, Taiwan ${ }^{4}$ Department of Health Industry Management, School of Healthcare Management, Kainan University, Taoyuan, Taiwan

${ }^{5}$ School of Oral Hygiene, College of Oral Medicine, Taipei Medical University, Taipei, Taiwan

${ }^{6}$ Department of Health Care Management, College of Management, Chang Gung University, Taoyuan, Taiwan

Twitter Wen-Feng Hsu @Wen-Feng

Contributors Conception and design of the study: H-MC, Y-CL, Y-WW, M-SW and TH-HC. Generation, collection, assembly, analysis and/or interpretation of data: H-HJ, H-MC, AM-FY, W-FH, JC-YF, C-YH, Y-CJ, SY-HC and L-SC. Drafting and revision of the manuscript: $\mathrm{H}-\mathrm{MC}$ and $\mathrm{TH}-\mathrm{HC}$. Approval of the final version of the manuscript: all authors.

Funding This study was supported by the Health Promotion Administration, Ministry of Health and Welfare (A1011119, A1021227, A1031135, A1041122, A1051013 and A1061224) of the Taiwanese government and the Ministry of Science and Technology grant (MOST 108-2118-M-002-002-MY3). The funding source had no role in the study design, data collection, analysis, interpretation, report writing or the decision to submit this paper for publication.

Competing interests All authors have completed the ICMJE uniform disclosure form (http://www.icmje.org/coi_disclosure.pdf) and declare no support from any organisation for the submitted work, no financial relationships with any organisations that might have an interest in the submitted work in the previous 3 years and no other relationships or activities that could appear to have influenced the submitted work.

\section{Patient consent for publication Not required.}

Ethics approval The research ethics committee of National Taiwan University Hospital approved this project and granted a waiver for informed consent (202002091W) pursuant to the regulations of the institutional review board. The study protocol was reviewed and approved by the Health Promotion Administration of Taiwanese government.

Provenance and peer review Not commissioned; externally peer reviewed. Data availability statement Data are available upon reasonable request. Supplemental material This content has been supplied by the author(s). It has not been vetted by BMJ Publishing Group Limited (BMJ) and may not have been peer-reviewed. Any opinions or recommendations discussed are solely those of the author(s) and are not endorsed by BMJ. BMJ disclaims all liability and responsibility arising from any reliance placed on the content. Where the content includes any translated material, BMJ does not warrant the accuracy and reliability of the translations (including but not limited to local regulations, clinical guidelines, terminology, drug names and drug dosages), and is not responsible for any error and/or omissions arising from translation and adaptation or otherwise.

Open access This is an open access article distributed in accordance with the Creative Commons Attribution Non Commercial (CC BY-NC 4.0) license, which permits others to distribute, remix, adapt, build upon this work non-commercially, and license their derivative works on different terms, provided the original work is properly cited, appropriate credit is given, any changes made indicated, and the use is non-commercial. See: http://creativecommons.org/licenses/by-nc/4.0/.

\section{ORCID iDs}

Han-Mo Chiu http://orcid.org/0000-0003-2786-8056

Amy Ming-Fang Yen http://orcid.org/0000-0002-0628-8768

Sherry Yueh-Hsia Chiu http://orcid.org/0000-0002-7207-7088

Sam Li-Sheng Chen http://orcid.org/0000-0002-5799-6705

Yi-Chia Lee http://orcid.org/0000-0002-8160-1216

Ming-Shiang Wu http://orcid.org/0000-0001-5325-3974

Tony Hsiu-Hsi Chen http://orcid.org/0000-0002-5799-6705 


\section{REFERENCES}

1 Hewitson P, Glasziou P, Watson E, et al. Cochrane systematic review of colorectal cancer screening using the fecal occult blood test (Hemoccult): an update. Am J Gastroenterol 2008;103:1541-9.

2 van Rossum LG, van Rijn AF, Laheij RJ, et al. Random comparison of guaiac and immunochemical fecal occult blood tests for colorectal cancer in a screening population. Gastroenterology 2008;135:82-90.

3 Chiu H-M, Chen SL-S, Yen AM-F, et al. Effectiveness of fecal immunochemical testing in reducing colorectal cancer mortality from the one million Taiwanese screening program. Cancer 2015;121:3221-9.

4 Zorzi M, Fedeli U, Schievano E, et al. Impact on colorectal cancer mortality of screening programmes based on the faecal immunochemical test. Gut 2015;64:784-90.

5 Giorgi Rossi P, Vicentini M, Sacchettini C, et al. Impact of screening program on incidence of colorectal cancer: a cohort study in Italy. Am J Gastroenterol 2015:110:1359-66.

6 Levin TR, Corley DA, Jensen CD, et al. Effects of organized colorectal cancer screening on cancer incidence and mortality in a large community-based population. Gastroenterology 2018;155:1383-91.

7 NCD Countdown 2030 collaborators. Ncd countdown 2030: worldwide trends in noncommunicable disease mortality and progress towards sustainable development goal target 3.4. Lancet 2018;392:1072-88.

8 Quintero E, Castells A, Bujanda L, et al. Colonoscopy versus fecal immunochemical testing in colorectal-cancer screening. N Engl J Med 2012:366:697-706.

9 Zauber AG, Lansdorp-Vogelaar I, Knudsen AB, et al. Evaluating test strategies for colorectal cancer screening: a decision analysis for the U.S. preventive services Task force. Ann Intern Med 2008;149:659-69.

10 Knudsen $A B$, Zauber AG, Rutter CM, et al. Estimation of benefits, burden, and harms of colorectal cancer screening strategies: modeling study for the US preventive services Task force. JAMA 2016;315:2595-609.

11 Chiu H-M, Lee Y-C, Tu C-H, Lee Y-C, Tu C-H, et al. Association between early stage colon neoplasms and false-negative results from the fecal immunochemical test. Clin Gastroenterol Hepatol 2013;11:832-8.

12 Wong MCS, Ching JYL, Chan VCW, et al. Diagnostic Accuracy of a Qualitative Fecal Immunochemical Test Varies With Location of Neoplasia But Not Number of Specimens. Clin Gastroenterol Hepatol 2015;13:1472-9.

13 Chou C-K, Chen SL-S, Yen AM-F, et al. Outreach and inreach organized service screening programs for colorectal cancer. PLOS One 2016;11:e0155276.

14 Chiu SY-H, Chuang S-L, Chen SL-S, et al. Faecal haemoglobin concentration influences risk prediction of interval cancers resulting from inadequate colonoscopy quality: analysis of the Taiwanese nationwide colorectal cancer screening program. Gut 2017;66:293-300

15 Chiang T-H, Chuang S-L, Chen SL-S, et al. Difference in performance of fecal immunochemical tests with the same hemoglobin cutoff concentration in a nationwide colorectal cancer screening program. Gastroenterology 2014;147:1317-26

16 Chiang C-J, You S-L, Chen C-J, et al. Quality assessment and improvement of nationwide cancer registration system in Taiwan: a review. Jpn J Clin Oncol 2015;45:291-6.

17 Cuzick J, Edwards R, Segnan N. Adjusting for non-compliance and contamination in randomized clinical trials. Stat Med 1997;16:1017-29.

18 Duffy SW, Cuzick J, Tabar L, et al. Correcting for non-compliance bias in casecontrol studies to evaluate cancer screening programmes. J Roy Stat Soc C-App Stat 2002;51:235-43.
19 Wu JC-Y, Anttila A, Yen AM-F, et al. Evaluation of breast cancer service screening programme with a Bayesian approach: mortality analysis in a Finnish region. Breast Cancer Res Treat 2010;121:671-8.

20 Tabar L, Yen M-F, Vitak B, et al. Mammography service screening and mortality in breast cancer patients: 20-year follow-up before and after introduction of screening. Lancet 2003;361:1405-10.

21 le Clercq CMC, Bouwens MWE, Rondagh EJA, et al. Postcolonoscopy colorectal cancers are preventable: a population-based study. Gut 2014;63:957-63.

22 Baxter NN, Sutradhar R, Forbes SS, et al. Analysis of administrative data finds endoscopist quality measures associated with postcolonoscopy colorectal cancer. Gastroenterology 2011;140:65-72.

23 Kaminski MF, Regula J, Kraszewska E, et al. Quality indicators for colonoscopy and the risk of interval cancer. N Engl J Med 2010;362:1795-803.

24 Corley DA, Jensen CD, Marks AR, et al. Adenoma detection rate and risk of colorectal cancer and death. N Engl J Med 2014;370:1298-306.

25 Phipps Al, Limburg PJ, Baron JA, et al. Association between molecular subtypes of colorectal cancer and patient survival. Gastroenterology 2015;148:77-87.

26 Bläker H, Alwers E, Arnold A, et al. The association between mutations in BRAF and colorectal cancer-specific survival depends on microsatellite status and tumor stage. Clin Gastroenterol Hepatol 2019;17:455-62.

27 Chang L-C, Shun C-T, Hsu W-F, et al. Fecal Immunochemical Test Detects Sessile Serrated Adenomas and Polyps With a Low Level of Sensitivity. Clin Gastroenterol Hepatol 2017;15:872-9.

28 Goldschmiedt M, Ahlquist DA, Wieand HS, et al. Measurement of degraded fecal hemoglobin-heme to estimate gastrointestinal site of occult bleeding. appraisal of its clinical utility. Dig Dis Sci 1988;33:605-8.

29 Lee Y-C, Li-Sheng Chen S, Ming-Fang Yen A, et al. Association between colorectal cancer mortality and gradient fecal hemoglobin concentration in colonoscopy Noncompliers. J Nat/ Cancer Inst 2017;109:djw269. doi:10.1093/jnci/djw269

30 Zorzi M, Hassan C, Capodaglio G, et al. Divergent long-term detection rates of proximal and distal advanced neoplasia in fecal immunochemical test screening programs: a retrospective cohort study. Ann Intern Med 2018;169:602-9.

31 Chiu H-M, Lee Y-C, Chou C-kuang, et al. Mo1929 Comparison of One-Day and Two-Day Sampling on Detection of Significant Colorectal Neoplasm by Fecal Immunochemical Test - Preliminary Results From a Population-Based Randomized Controlled Trial. Gastroenterology 2015;148:S742.

32 Imperiale TF, Ransohoff DF, Itzkowitz SH, et al. Multitarget stool DNA testing for colorectal-cancer screening. N Engl J Med 2014;370:1287-97.

33 Jen $\mathrm{H}-\mathrm{H}$, Hsu C-Y, Chen SL-S, et al. Rolling-out screening volume affecting compliance rate and waiting time of FIT-based colonoscopy. J Clin Gastroenterol 2018;52:821-7.

34 Issaka RB, Singh MH, Oshima SM, et al. Inadequate utilization of diagnostic colonoscopy following abnormal fit results in an integrated safety-net system. Am J Gastroenterol 2017:112:375-82.

35 Martin J, Halm EA, Tiro JA, et al. Reasons for lack of diagnostic colonoscopy after positive result on fecal immunochemical test in a safety-net health system. Am J Med 2017:130:93.e1-93.e7.

36 Chen C-D, Yen M-F, Wang W-M, et al. A case-cohort study for the disease natural history of adenoma-carcinoma and de novo carcinoma and surveillance of colon and rectum after polypectomy: implication for efficacy of colonoscopy. Br J Cancer 2003:88:1866-73.

37 Yang KC, Liao CS, Chiu YH. Colorectal cancer screening with fecal occult blood test within a multiple disease screening program: an experience from Keelung, Taiwan. $J$ Med Screen 2006;13:8-13.

38 Mandel JS, Church TR, Bond JH, et al. The effect of fecal occult-blood screening on the incidence of colorectal cancer. N Engl J Med 2000;343:1603-7. 